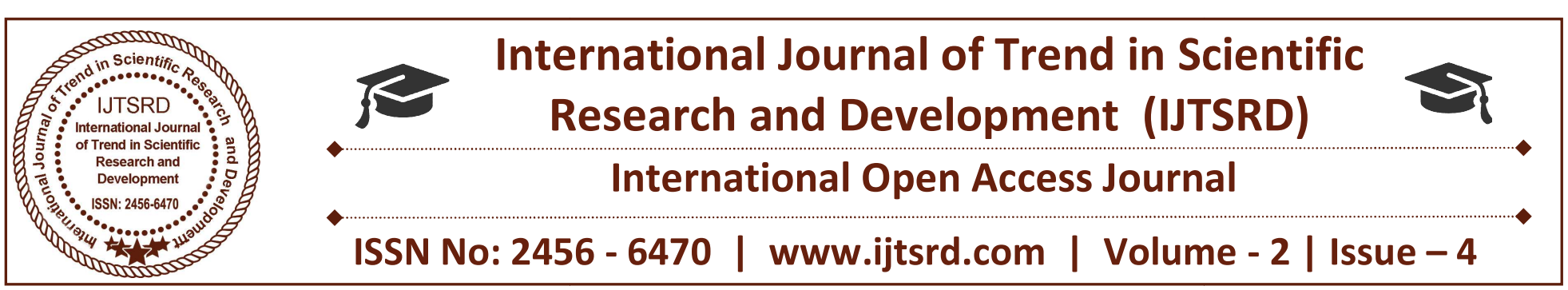

\title{
A Review - Study of the Thermo-Physical Properties of PCM with Dispersion of Nano Particles
}

\author{
Vivek Baghel ${ }^{1}$, Yogesh Yadav \\ ${ }^{1}$ M.Tech Scholar, ${ }^{2}$ Assistant Professor \\ Millennium Institute of Technology, Bhopal, Madhya Pradesh, India
}

\begin{abstract}
In this article, a new sort of nanofluid phase change materials (PCMs) is developed by suspending a small amount of $\mathrm{BeO}$ nanoparticles in solution of wax. Its thermal conductivities, super cooling degree, latent heat, specific heat, and rheological behaviours of the nanofluids PCMs have investigated. A rectangular duct designed and numerical studies were performed to reveal the effects of molten PCM thermo physical properties on heat and exergy storage performance of latent heat storage (LHS) unit. The range-analysis, regression analysis and optimization were conducted. The PCM selection criteria were established. The results show that for short time LHS system, the order of PCM properties effects on heat storage performance is melting temperature, thermal conductivity, specific heat, density and melting enthalpy successively. PCM with lower melting temperature, higher thermal conductivity and specific heat is beneficial to improve the heat storage rate and heat storage quality. For long time heat storage system, the order of PCM properties effects on heat storage performance is density, melting enthalpy, specific heat, melting temperature and thermal conductivity successively. PCM with higher density, melting enthalpy and specific heat can both improve the heat storage rate and heat storage quality.
\end{abstract}

Keywords: CFD, Nanofluids, Phase change material (PCM) Thermophysical properties, Cool storage

\section{INTRODUCTION}

Energy conservation and environment protection are the very important topics in the field of energy. Cool thermal energy storage has become one of the primary solutions to the electrical power imbalance between

daytime need and night time abundance. Phase change materials (PCMs) are a kind of very important latent heat energy storage materials. It has wide applications in many fields. Such as refrigeration and air conditioning systems, solar energy systems, and heating and cooling of buildings. It has attracted great interest in recent years. Inorganic compounds are the one of major categories PCMs used in building cool storage applications. Most inorganic PCMs such as salt hydrates are chemically stable and non-ignitable. However, the problems of bigger super cooling degree and poor thermal conductivity exist. The big super cooling degree makes the evaporating temperature of refrigerator lower, and reduces the refrigeration efficiency. In addition, the low thermal conductivity unable to enhance the performance of energy storage devices. Numerous investigations have been made to solve these thermal properties limitation. On the one hand, in order to eliminate the super cooling degree, many researchers used ultrasonic vibration or add some chemistry nucleating agents.

Literature Review: In recent years, a nanofluid has been employed to enhance the heat transfer in PCMs for energy storage, due to its desirable properties of high thermal conductivity.

M.Cheralathan et al.[2006] Investigated the transient behavior of a phase change material based cool thermal energy storage (CTES) system comprised of a cylindrical storage tank filled with encapsulated phase change materials (PCMs) in spherical container integrated with an ethylene glycol chiller plant. A simulation program was developed to evaluate the temperature histories of the heat transfer fluid (HTF) 
and the phase change material at any axial location during the charging period. The results of the model were validated by comparison with experimental results of temperature profiles of HTF and PCM. The results showed that increase in porosity contributes to a higher rate of energy storage.

Nayak A.O. et al. [2011] He has considered various PCM like paraffin wax, sodium acetate tri-hydrate and phenolphthalein which are used to absorb heat from the coolant water from the engine. Due to conduction and convection of heat transfer heat is stored inside the PCM in the form of latent heat. Convection and heat flux effect due to temperature change has been simulated and studied in detail using GAMBIT and FLUENT. From the temperature profiles obtained from the analysis he concluded that sodium acetate tri-hydrate gives us the most promising results as compared to paraffin wax and naphthalene. Coolant water loses maximum heat to sodium acetate trihydrate which is obtained as drop in temperature from $343 \mathrm{~K}$ to $324 \mathrm{~K}$ (in the coolant water). He observed that the heat absorption in the PCM material decreases gradually as it travels from the inlet of coolant water towards the outlet of coolant water.

Bauer T. et al. [2012] this paper focuses on latent heat storage using a phase change material (PCM). The paper lists of literature and gives the current status of medium working range temperature of 200 to $350 \mathrm{oC}$. In this paper the system with KNO3-NaNO3 is discussed in detail with their thermo-physical properties in the liquid and solid phase. A comparison of literature data and own measurements for the density, heat capacity, thermal diffusivity and thermal conductivity is presented in detail. The melting temperature and enthalpy of the KNO3- NaNO3 is $222 \mathrm{oC}$ and $108 \mathrm{j} / \mathrm{g}$ was identified respectively. Different properties such as thermal conductivity, density are also collected from the different literatures.

Chieruzzi M. et al. [2013]: In this study, binary salt NaNO3- KNO3 (60:40 ratio) is used to prepare the different nano fluid. Measurements on thermo physical properties were performed by differential scanning calorimetry analysis and the dispersion of the nanoparticles was analyzed by scanning electron microscopy (SEM). The nanoparticles used were silica ( $\mathrm{SiO} 2)$, alumina (A12O3), titania ( $\mathrm{TiO} 2)$, and a mix of silica-alumina ( $\mathrm{SiO} 2-\mathrm{Al} 2 \mathrm{O} 3)$. Three weight fractions $0.5,1.0$, and $1.5 \%$ by weight are selected for the experimentation work. Each nano fluid was firstly prepared in water solution then it is sonicated and evaporated. The results from the experiments show that the addition of $1.0 \mathrm{wt} . \%$ of silica and aluminium oxide nanoparticles to the base salt increases the specific heat $15 \%$ to $57 \%$ in the solid phase and of $1 \%$ to $22 \%$ in the liquid phase. There is also decrease in melting point by $80 \mathrm{C}$ for addition of $1.0 \%$ of $\mathrm{SiO} 2$ and $\mathrm{A} 12 \mathrm{O} 3$ nanoparticles. These nanofluids can be used in concentrating solar plants with a reduction of storage material due to improvement in the specific heat is achieved.

Dudha B. et al [2013]. In this study, eutectic of sodium nitrate and potassium nitrate at $60: 40$ by weight were chosen as the base molten salt and silica nanoparticles were used to enhance the specific heat capacity of the salts. A modulated differential scanning calorimeter was employed to measure the specific heat capacity. He used sizes 5, 10, 30 and 60 $\mathrm{nm}$ of nanoparticles for the investigation of effect of size of the nanoparticle on the specific heat capacity. It was seen that the doping of nanoparticles enhanced the specific heat capacity by approximately $27 \%$ for $60 \mathrm{~nm}$. Material characterization is carried out with the help of the Scanning Electron Microscope (SEM) to explore the cause of the enhanced specific heat capacity. It was observed that as the amount of nanostructures increases the enhancement of specific heat capacity also increasesA large number of phase change materials (organic, inorganic and eutectic) are available in any required temperature range. There are a large number of organic and inorganic chemical materials, which can be identified as PCM from the point of view melting temperature and latent heat of fusion. However, except for the melting point in the operating range, majority of phase change materials does not satisfy the criteria required for an adequate storage media as discussed earlier. As no single material can have all the required properties for an ideal thermal-storage media, one has to use the available materials and try to make up for the poor physical property by an adequate system design.

The most important thermo physical properties such as thermal conductivity, latent heat of phase change, specific heat, degree of super cooling and viscosity were investigated by using computational fluid dynamics. The aim is to analyze the effects of volume fraction of the $\mathrm{BeO}$ nanoparticles suspended into nanofluids PCMs. 
Methodology: In this study a CFD model of rectangular block has been design in ANSYS Fluent 14.5 by using design modular which has been shown in figure below. The meshing of the model will be done in ICEM CFD Meshing. The numbers of nodes and the elements have been defined after then the name selection will be done and the solution of the problem will solve on the CFD post processing by using desired Boundary conditions.

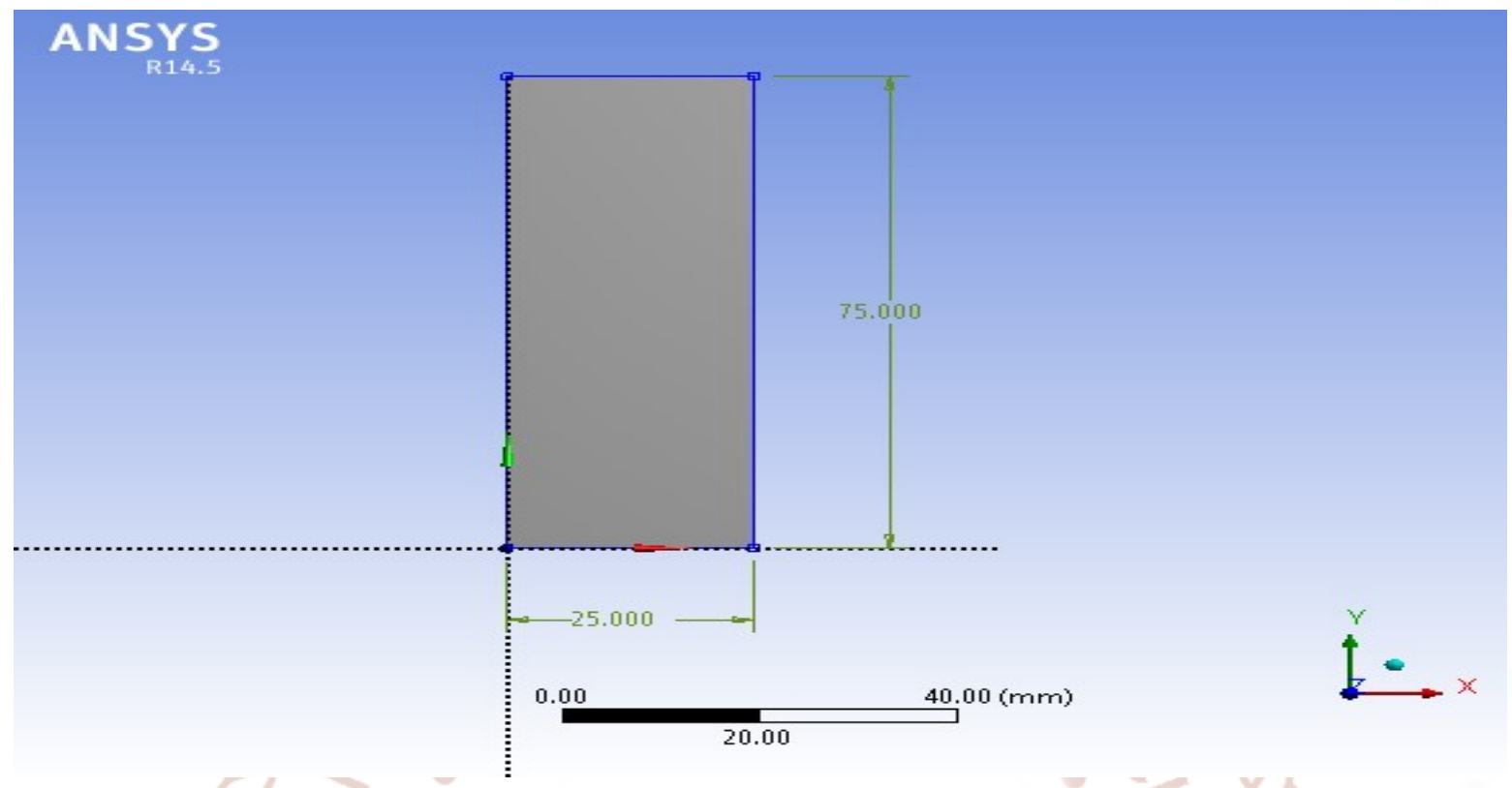

Figure 1 Wall of the paraffin wax of rectangular enclosure $25 \mathrm{~mm} * 75 \mathrm{~mm}$

Conclusion: In this analysis, the cumulative effect on melting rate and thermo-physical properties in phase change material will be investigate using CFD analysis. Based on the results obtain by the CFD and mathematical calculations it will found that the Thermophysical properties of phase change materials will improve. It can be recommended that the low temperature $\mathrm{BeO}$ nanofluids PCMs are suitable for the industries low temperature energy storage.

\section{References:}

1) Kalaiselvam S, Parameshwaran R, Harikrishnan $S$. Analytical and experimental investigations of nanoparticles embedded phase change materials for cooling application in modern buildings. Renew Energy 2012; 39:375-87.

2) Alkan C, Sarı A, Karaipekli A. Preparation, thermal properties and thermal reliability of microencapsulated n-eicosane as novel phase change material for thermal energy storage. Energy Convers Manage 2011; 52:687-92.

3) Liu Z, Zhu Q. Application of aqueous nanofluids in a horizontal mesh heat pipe. Energy Convers Manage 2011; 52:292-300.
4) Tang Zh, Liu A, Chen Zh. Study on performance of colloidal mixtures consisted of stearic acid and $\mathrm{Na} 2 \mathrm{HPO} 412 \mathrm{H} 2 \mathrm{O}$ for use as phase change materials of thermal energy storage. Energy Convers Manage 2010; 51:1459-63.

5) - Okawa S, Saito A, Kadoma Y, Kumano H. Study on a method to induce freezing of super cooled solution using a membrane. Int J Refrig 2010; 33:1459-64.

6) Medrano M, Yilmaz MO, Nogues M. Experimental evaluation of commercial heat exchangers for use as PCM thermal storage systems. Appl Energy 2009; 86:2047-55.

7) Shukla A, Buddhi D, Sawhney RL. Solar water heaters with phase change material thermal energy storage medium: a review. Renew Sustain Energy Rev 2009; 13:2119-25.

8) Lin Y, Kang S, Chen H. Effect of silver nanofluid on pulsating heat pipe thermal performance. Appl Therm Eng 2008; 28:1312-7.

9) Cheralathan $M$, Velraj $R$, Renganarayanan $S$. Performance analysis on industrial refrigeration system integrated with encapsulated PCM-based cool thermal energy storage system. Int J Energy Res 2007; 31:1398-413. 
10) $\mathrm{Su}$ JC, Liu PS. A novel solid-solid phase change heat storage materials with polyurethane block copolymer structure. Energy Convers Manage 2006; 47:3185-91.

11) Hamada Y, Otsu W, Fukai J, Morozumi Y, Miyatake O. Anisotropic heat transfer in composites based on high-thermal conductive carbon fibers. Energy 2005; 30:221-33.

12) Xiao M, Feng B, Gong K. Preparation and performance of shape stabilized phase change thermal storage materials with high thermal conductivity. Energy Convers Manage 2002; 43:103-8.

13) Okawa S, Saito A, Minami R. The solidification phenomenon of the super cooled water containing solid particles. Int J Refrig 2001; 24:108-17.

14) Lee S, Choi SUS. Application of metallic nanoparticles suspensions in advanced cooling system. In: Kwon Y, Davis D, Chang H, editors. Recent advance in solids/structures and application of metallic materials. Vol 72. New York: ASME; 1996. p. 227-34.

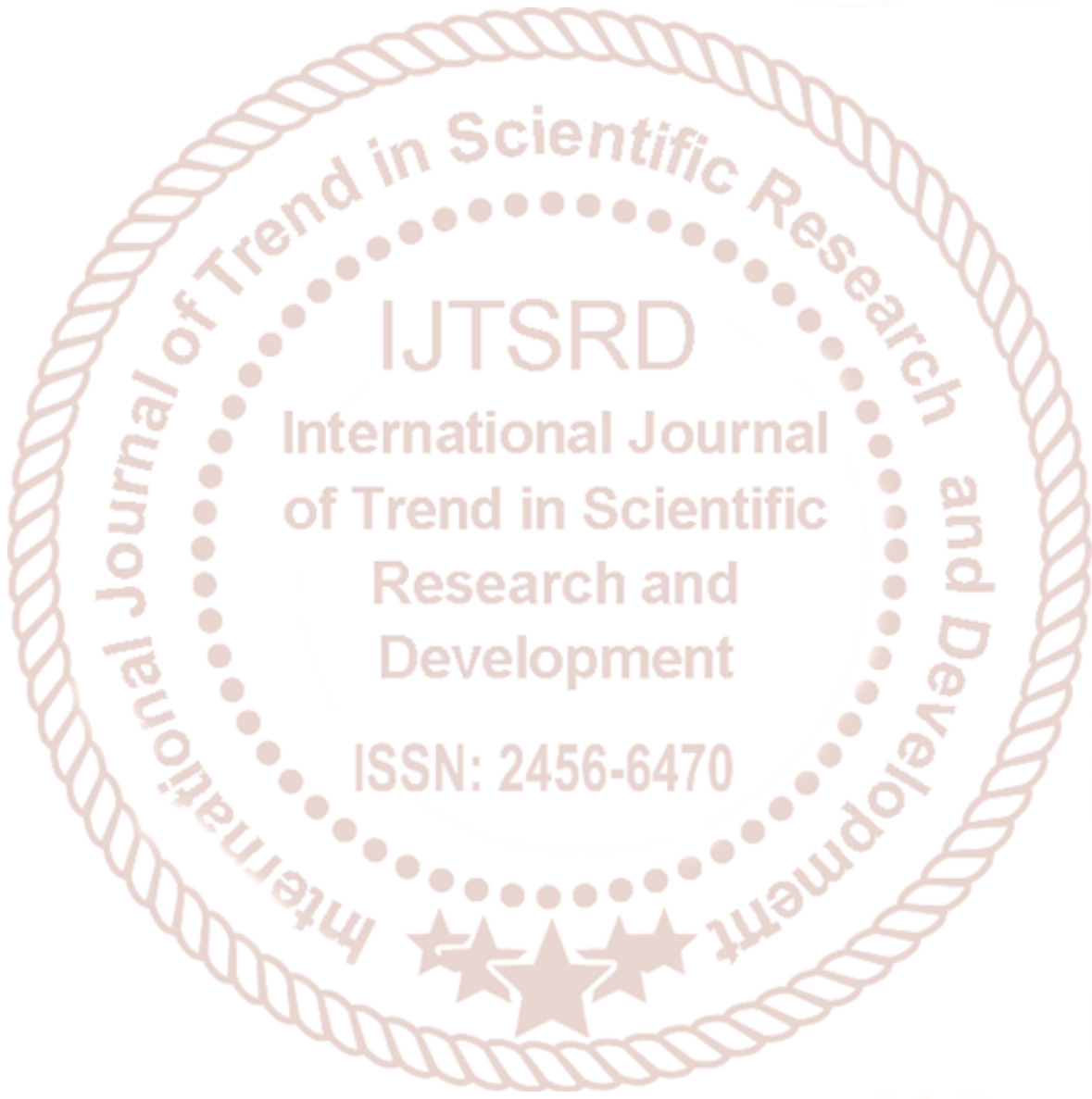

